\title{
Exploring gene function and parasite-host protein interactions
}

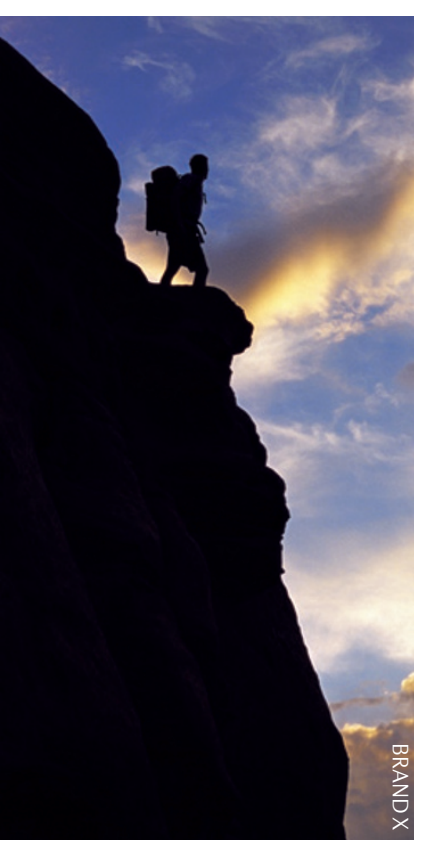

Two new studies present methods to explore how host and parasite transcriptomes interact with one another. Yamagishi and colleagues describe a new approach to analyse human and parasite RNA expression profiles simultaneously, and Lisewski and colleagues demonstrate the feasibility of compressing biological networks by eliminating redundant evolutionary relationships to enable functional predictions.

With the aim of better understanding the ways in which the transcriptomes of hosts and parasites interact with each other, Yamagishi and colleagues extracted peripheral blood (which contains a mixture of both host and parasite transcripts) from 116 Indonesian individuals infected with Plasmodium falciparum. After extracting and sequencing the RNA,
RNA sequencing (RNA-seq) tags were mapped back to the reference genomes. Notably, the team identified pairs of human and parasite genes that are correlated, and some of these correlations are enriched in particular gene ontology categories, including the innate immune response in humans and metabolic processes in the parasite. However, this interpretation was hindered by the lack of gene function information for the P. falciparum genes $-44 \%$ of these RNA-seq tags were derived from functionally uncharacterized genes.

Computational network analyses are often used to infer gene functions, although these methods are limited in their capacity to infer function in sparsely annotated genomes, including the $P$. falciparum genome, and are computationally demanding. Lisewski and colleagues developed a compression approach to reduce the number of links within a 'supergenomic' network and applied this method to determine the molecular function of EXP1, which is an abundantly transcribed $P$. falciparum gene. From this approach, the team predicted that EXP1 is a core component of glutathione metabolism, and they were then able to use this information to experimentally validate that EXP1 is a glutathione $S$-transferase that conjugates glutathione onto haematin (the main cytotoxin released during malarial blood stage infection).

Taken together, these papers provide insights into host-parasite transcriptome interactions, which will be key to developing effective treatment strategies for malaria in the future.

Bryony Jones

ORIGINAL RESEARCH PAPERS Yamagishi, J. et al. Interactive transcriptome analysis of malaria patients and infecting Plasmodium falciparum.

Genome Res. http://dx.doi.org/10.1101/ gr.158980.113 (2014) | Lisewski, A. M. et al. Supergenomic network compression and the discovery of EXP1 as a glutathione transferase inhibited by artesunate. Cell 158, 916-928 (2014) 